\title{
Development of Environmental-Friendly Biodegradable Polymers with Antibacterial Properties
}

\author{
R. Sakuma, K. Sawada, K. Nakashima, T. Tsukegi, S. Osawa, and O. Yoshimura \\ Kanazawa Institute of Technology, 3-1 Yatsukaho, Hakusan, Ishikawa 924-0838 Japan \\ E-mail: b1324233@planet.kanazawa-it.ac.jp
}

\begin{abstract}
In this study, a novel antibacterial polymeric material with low environmental impact was produced by using cellulose acetate butyrate (CAB), a biodegradable plastic, and hiba oil, a natural antibacterial agent. The method of material preparation, antibacterial performance, and mechanical properties were evaluated. When the film was prepared using acetone, hiba oil did not disperse, and a white film was produced. When the film was prepared using chloroform, which is not miscible with water, hiba oil was uniformly dispersed in $\mathrm{CAB}$, and a transparent film was produced. Antibacterial performance was obtained by the inclusion of hiba oil at $20 \mathrm{wt} \%$ of CAB. In addition, hiba oil conferred flexibility to $\mathrm{CAB}$ through its action as a plasticizer.

Key words: cellulose acetate butyrate, hiba oil, antibacterial, miscibility, dispersible
\end{abstract}

\section{INTRODUCTION}

Since the 1990s, environments suitable for bacterial and fungal growth have increased owing to an increase in moisture accompanying the high airtightness of houses and insufficient ventilation[1-2]. In addition, bacterial problems, such as mass food poisoning by Escherichia coli O-157 and Legionella pneumophila in 24 hour baths, are occurring with increasing frequency. Owing to these problems, the requirement for cleanliness and safety has increased among consumers. Consequently, the market for antibacterial plastics has expanded, particularly for kitchen, toilet, and bath products.

In general, plastics such as polyethylene (PE) and polypropylene (PP), which are widely used as a products for base materials, offer advantages such as low price, light weight, easy molding, and durability[3]. For these reasons, PE and PP are used for many products. However, it is hard for these products to decompose in the natural environment owing to their durability. Disposal of plastics may occur through recycling (30\%), landfill (20\%), and incineration (50\%)[4]. Therefore, problems may occur when plastics that are difficult to decompose are left in the environment as landfill; the disposal of these plastics is a burden on the environment. Consequently, antibacterial polymers with lower environmental impact are desired.

In this research, we have focused on biodegradable plastics with low environmental burden and natural antibacterial agents with suitability volatility. In nature, biodegradable plastics are decomposed by microorganisms after use and converted into water and carbon dioxide[5]. The natural antibacterial agent in this study focused on hiba oil. Hiba oil, an extract from hiba wood, is able to suppress the growth of bacteria at much lower concentrations than other wood extract oils[6-7]. In addition, it contains hinokitiol, which has an excellent antibacterial effect. It is known that hinokitiol has a broad antibacterial spectrum and resistance to hinokitiol is less likely to occur. In addition, as hinokitiol is volati, it hardly remains in the material at disposal. In contrast, inorganic or synthetic organic types of antibacterial agents do not disappear from the material. Therefore, after the biodegradable plastic is decomposed, their antibacterials remain in the environment.
Therefore, we aimed to develop an antibacterial polymeric material with less environmental burden by using biodegradable plastic and natural antibacterial agents.

\section{EXPERIMENTAL}

\subsection{Materials}

Cellulose acetate butyrate (CAB, butyryl content $35 \%$ $39 \%$, Acaros Organics) was used as the biodegradable plastic. It is readily soluble in organic solvents and can be molded without using heat[8-9]. Hiba oil (Aomorihiba) was used as the natural antibacterial agent. Escherichia coli ( $E$. coli) JM 109 (Nippon Gene) was used as the test strain.

2.2 Preparation of CAB film containing hiba oil (at $10 \mathrm{wt} \%$ hiba oil)

Hiba oil $(0.1 \mathrm{~g})$ was mixed with CAB $(0.9 \mathrm{~g})$ in acetone $(30 \mathrm{~mL})$. The mixture was shaken until dissolution, and defoamed under reduced pressure with a desiccator, after which films were obtained by the casting method.

\subsection{Halo test of $\mathrm{CAB}$ film containing hiba oil}

E. coli was cultured in Nutrient Broth (NB) medium at $37{ }^{\circ} \mathrm{C}$ for $24 \mathrm{~h}[10]$. The E. coli culture was diluted from $1.0 \times 10^{6}$ cells $/ \mathrm{mL}$ to $1.0 \times 10^{7}$ cells $/ \mathrm{mL}$ in NB medium. $E$. coli culture $(100 \mu \mathrm{L})$ prepared bacterial count was smeared on Nutrient Agar (NA) medium. CAB film containing hiba oil ( $\Phi 10 \mathrm{~mm}$ ) was placed in the center of the NA medium and incubated at $37^{\circ} \mathrm{C}$ for $24 \mathrm{~h}$ in a thermostatic incubator.

\subsection{Shaking test of $\mathrm{CAB}$ film containing hiba oil}

E. coli was cultured in NA medium at $35^{\circ} \mathrm{C}$ for $20 \mathrm{~h}[11]$. Bacterial suspensions $\left(1.0 \times 10^{4} \sim 5.0 \times 10^{4}\right.$ cells $\left./ \mathrm{mL}\right)$ were prepared in $1 / 500 \mathrm{NB}$ medium, and $10 \mathrm{~mL}$ was added to a $100 \mathrm{~mL}$ Erlenmeyer flask. CAB film $\left(2.0 \times 2.0 \mathrm{~cm}^{2}, 4\right.$ pieces) $\mathrm{CAB}$ film containing hiba oil $\left(2.0 \times 2.0 \mathrm{~cm}^{2}, 4\right.$ pieces) were added to each flask and shaken at $35{ }^{\circ} \mathrm{C}$ and $150 \mathrm{rpm}$ for $24 \mathrm{~h}$. Based on the bacterial fluid collected from each flask, a 10-fold dilution series was prepared with $1 / 500$ NB medium. A 10-fold dilution series was smeared on Standard Methods Agar (SA) medium and was cultivated at $35{ }^{\circ} \mathrm{C}$ for $48 \mathrm{~h}$ in a thermostatic incubator. 
After cultivation, the number of colonies was counted and the antibacterial activity value was calculated. The obtained antibacterial activity value was compared with the antibacterial performance criteria and the antibacterial performance was evaluated.

\subsection{Tensile test of $\mathrm{CAB}$ film containing hiba oil}

The tensile test was performed in accordance with the JIS K 7127 method[12]. CAB film and CAB film containing hiba oil were cut into strip form $\left(5 \times 50 \mathrm{~mm}^{2}\right)$. Both ends of the film were sandwiched by Tensilon (RTC-1225A, Orientec Co.) with a chuck distance of $30 \mathrm{~mm}$. In addition, waterproof paper \#1200 was interposed as a nonslip layer between the Tensilon and the sample. The tensile test was conducted at a tensile speed $3 \mathrm{~mm} / \mathrm{min}$. From the results, a Stress-Strain (S-S) curve was prepared and the strain, modulus of elasticity, and maximum stress were calculated.

2.6 Film preparation using chloroform and characterization The solvent was changed from acetone to chloroform, and the film was prepared by using the same method described in Section 2.2. In addition, surface observations, a halo test, a shaking test, and a tensile test of the produced film were performed. The results in chloroform and acetone were compared and the difference in properties induced by each solvent was evaluated.

\section{RESULTS AND DISCUSSION}

3.1 Halo test $\mathrm{CAB}$ film containing hiba oil

The results of the halo test on $\mathrm{CAB}$ film containing hiba oil are shown in Fig. 1. The CAB film containing $10 \mathrm{wt} \%$ hiba oil did not show antibacterial activity. However, as a halo was observed after from CAB film containing $20 \mathrm{wt} \%$ hiba oil, the antibacterial properties were confirmed. In addition, it became clear that it was necessary to add hiba oil at $20 \mathrm{wt} \%$ in order to impart antibacterial properties to the material.

\subsection{Shaking test $\mathrm{CAB}$ film containing hiba oil}

The results of the antibacterial activity were calculated based on the results from the shaking test. The antibacterial activity values of $\mathrm{CAB}$ film containing $20 \mathrm{wt} \%$ and $30 \mathrm{wt} \%$ hiba oil were 1.0 and 4.3 , respectively. The antibacterial performance criteria of antibacterial products is 2.0 ; thus, the $\mathrm{CAB}$ film containing $30 \mathrm{wt} \%$ hiba oil exceeded this criteria[13], and it was suggested that it has sufficient antibacterial performance.

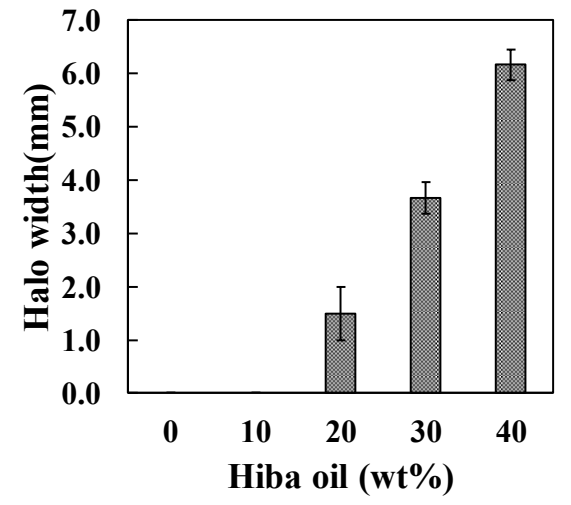

Fig. 1. Halo test results of $\mathrm{CAB}$ film containing hiba oil

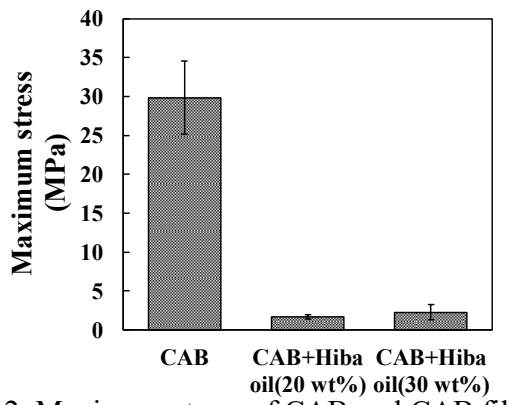

Fig. 2. Maximum stress of $\mathrm{CAB}$ and $\mathrm{CAB}$ film containing hiba oil

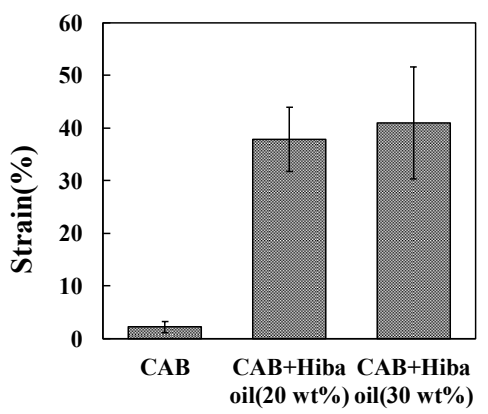

Fig. 3. Strain of $\mathrm{CAB}$ and $\mathrm{CAB}$ film containing hiba oil

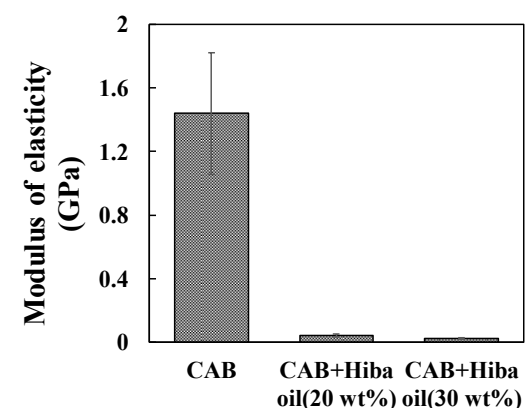

Fig. 4. Modulus of elasticity of $\mathrm{CAB}$ and $\mathrm{CAB}$ film containing hiba oil

3.3 Tensile test of CAB film containing hiba oil

The mechanical properties obtained from the tensile test are shown in Fig. 2-4. The relative strain was improved 17 times or more by the addition of $20 \mathrm{wt} \%$ hiba oil to CAB. Thus, it was confirmed that the modulus of elasticity was reduced, most likely because hiba oil functioned as a plasticizer. Hiba oil contains two components, an acidic oil composed of phenols and a neutral oil composed of sesquiterpenes[14-15]. The sesquiterpenes present in this neutral oil are used as polymer modifiers and tackifiers[16], which are used to impart fluidity to the base material[17]. Therefore, it was suggested that $\mathrm{CAB}$ was plasticized by the addition of hiba oil, and that flexibility was imparted to $\mathrm{CAB}$ by the addition of hiba oil.

On the other hand, with this increased flexibility, the maximum stress was remarkably decreased. In the $\mathrm{CAB}$ film containing hiba oil prepared under the conditions described in Section 2.3, the transparent portion and the white portion were mixed (Fig. 5). This mixed state is considered to be related to remarkable decrease in the maximum stress. Therefore, the surface of the film was observed by using SEM. 


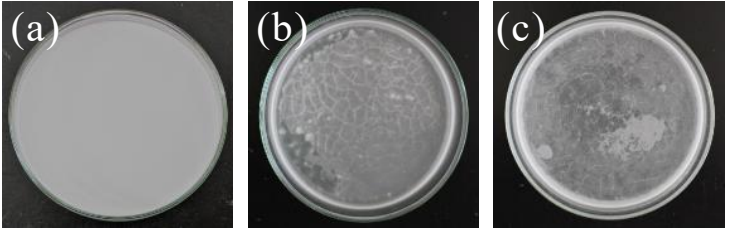

Fig. 5 CAB film prepared using acetone. (a) $\mathrm{CAB}$ (b) $\mathrm{CAB}$ containing $20 \mathrm{wt} \%$ hiba oil (c) $\mathrm{CAB}$ containing $30 \mathrm{wt} \%$ hiba oil

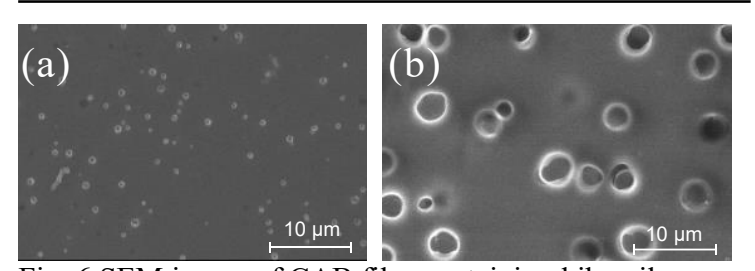

Fig. 6 SEM image of CAB film containing hiba oil prepared using acetone. (a) $20 \mathrm{wt} \%$ hiba oil (b) $30 \mathrm{wt} \%$ hiba oil
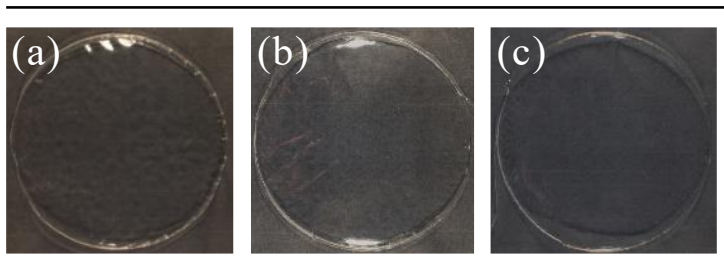

Fig. $7 \mathrm{CAB}$ film prepared using chloroform. (a) $\mathrm{CAB}$ (b) $\mathrm{CAB}$ containing $20 \mathrm{wt} \%$ hiba oil (c) $\mathrm{CAB}$ containing $30 \mathrm{wt} \%$ hiba oil

\subsection{Surface observation of film}

The SEM images of the CAB film containing $20 \mathrm{wt} \%$ and $30 \mathrm{wt} \%$ hiba oil are shown (Fig. 6). Round particles were observed in the white part of the $\mathrm{CAB}$ film containing hiba oil. In addition, as the amount of hiba oil increased, the particle size appeared to increase from approximately 0.5 $\mu \mathrm{m}$ to $3-5 \mu \mathrm{m}$, and thus these particles appear to be hiba oil. It was thought that the decrease in dispersibility was because acetone is a solvent with high miscibility with water. When a film is made with acetone, the moisture in the air is absorbed by acetone becomes mixed into the film.

Therefore, it was suggested that hiba oil and moisture repel each other, leading to discrete particles. Thus, films were prepared by using chloroform immiscible with water (Fig. 7). All films were confirmed to be transparent, which suggested that by using chloroform, moisture was excluded from the film, and hiba oil was uniformly dispersed.

\subsection{Antibacterial test of film using chloroform}

The results of the halo test on CAB film prepared using chloroform are shown in Fig. 8. Similar to the results for the films prepared with acetone, the presence of a halo was observed in $\mathrm{CAB}$ film containing $20 \mathrm{wt} \%$ hiba oil. This result confirmed that the antibacterial property was imparted even with chloroform.

To evaluate whether this film had antibacterial performance suitable for an antibacterial products, a shaking test was performed. The antibacterial activity values of the CAM film containing $20 \mathrm{wt} \%$ and $30 \mathrm{wt} \%$ hiba oil were 3.2 and 3.3, respectively. Both formulations exceeded the antibacterial performance criteria and fulfilled the conditions required of antibacterial products.

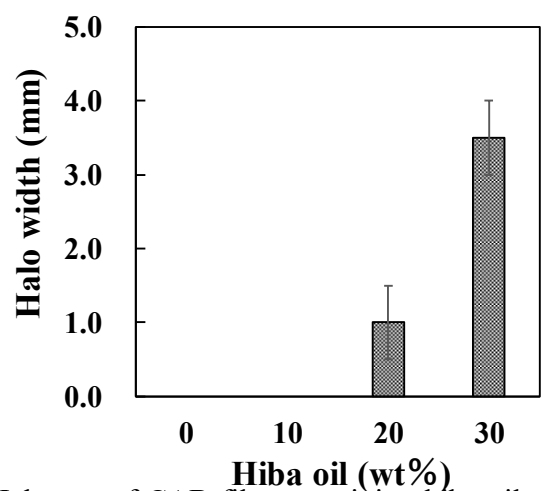

Fig. 8 Halo test of CAB film containing hiba oil prepared by using chloroform

In addition, as described above, in the case of films prepared using acetone, antibacterial performance was not obtained when $\mathrm{CAB}$ films were prepared containing 20 wt $\%$ hiba oil, but was obtained in the film prepared using chloroform. This was considered to be related to the concentration of hiba oil present on the film surface. As the antibacterial activity occurs through the contact of the antibacterial agent with bacteria, it is necessary for the antibacterial agent to be exposed on the surface[18]. It is suggested from this result that the amount of hiba oil on the film surface prepared with acetone was less than that prepared with chloroform. It is considered that dispersibility improves when prepared with chloroform.

3.6 Tensile test of film using chloroform

The mechanical properties obtained from the tensile test of films prepared by using acetone and chloroform are shown in Fig. 9-11. The films made using chloroform had better physical properties than those made using acetone. Furthermore, when prepared by using acetone, the maximum stress decreased by approximately $95 \%$ by the addition of hiba oil, whereas in the films prepared by using chloroform, the maximum stress only decreased by approximately $30 \%$. In addition, the strain of the film containing hiba oil was higher by $150 \%$ or more in the film prepared with chloroform. It is considered that the physical properties were improved by dispersing hiba oil in the film. In addition, $\mathrm{CAB}$ film prepared by using acetone was hard and brittle, but the film prepared by using chloroform was a flexible material. This difference was considered to be related to the incorporation of moisture when $\mathrm{CAB}$ was dissolved. The $\mathrm{CAB}$ film prepared by using acetone was

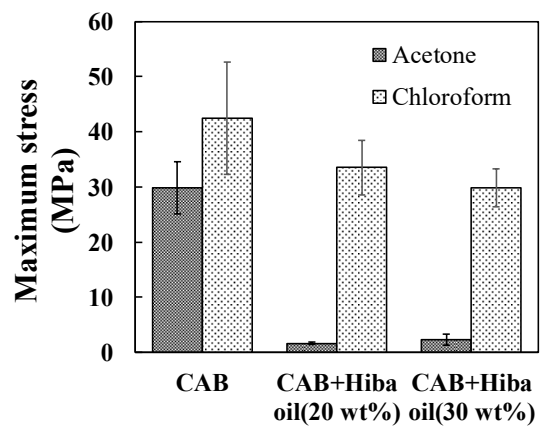

Fig. 9. Maximum stress of $\mathrm{CAB}$ and $\mathrm{CAB}$ film containing hiba oil prepared by using chloroform 


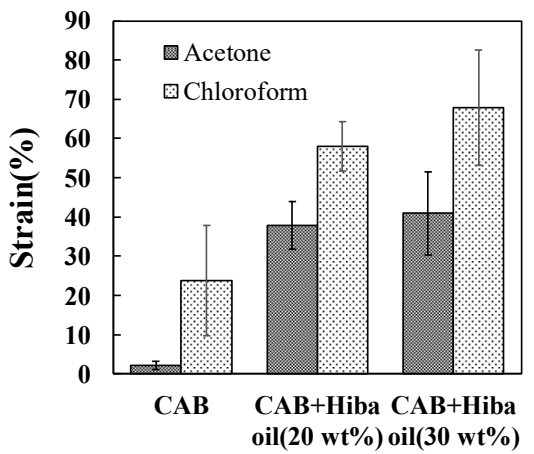

Fig. 10. Strain of $\mathrm{CAB}$ and $\mathrm{CAB}$ film containing hiba oil prepared by using chloroform

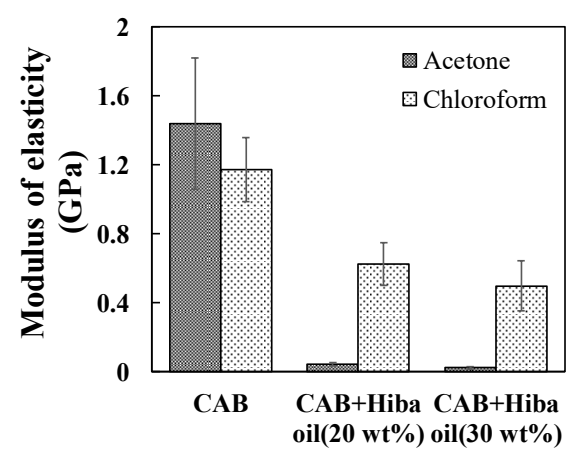

Fig. 11. Modulus of elasticity of $\mathrm{CAB}$ and $\mathrm{CAB}$ film containing hiba oil prepared by using chloroform

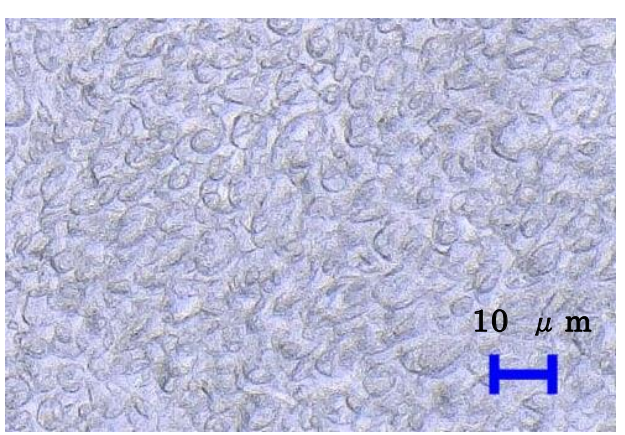

Fig. 12 Surface morphology of CAB film prepared by using acetone

observed by using a digital microscope (VHX-6000, Keyence Co.). A large number of holes was present on the film surface (Fig. 12). CAB is an acetylated and butyrylated part of the hydroxyl group of cellulose, so it is insoluble in water like cellulose. It is considered thought that $\mathrm{CAB}$ is not dissolved in water mixed in the solvent, but is dissolved only in acetone. Therefore, after the solvent volatilized, it was suggested that a cavity was formed in the place where water had been present, and that this cavity caused the deterioration of physical properties.

\section{CONCLUSION}

In this study, a $\mathrm{CAB}$ film containing hiba oil was produced in order to reduce the burden on the environment, and the examination of the antibacterial activity, mechanical properties, and production method of the film yielded the following results:

When $\mathrm{CAB}$ film containing hiba oil was prepared using acetone, $20 \mathrm{wt} \%$ hiba oil showed antibacterial properties in the halo test, and $30 \mathrm{wt} \%$ hiba oil satisfied the criteria in the shaking test. In the tensile test, flexibility was improved by the addition of hiba oil, but the maximum stress remarkably decreased.

When the films were prepared using chloroform, $20 \mathrm{wt} \%$ hiba oil satisfied the criteria in the shaking test. In the tensile test, hiba oil was uniformly dispersed, and the decrease in maximum stress was suppressed.

The SEM observations of the surface revealed that the dispersibility was higher in films prepared using chloroform than in films prepared by using acetone. In addition, it was suggested that through the use chloroform, which is not miscible with water, it was possible to prevent the water-induced deterioration of the physical properties of CAB. Even with antibacterial activity, the concentration of hiba oil present on the surface increased owing to the improvements in dispersibility, which suggested that the antibacterial activity was improved.

The above results indicate that a biodegradable polymer material with antibacterial properties can be prepared. Application of this material is a subject to be examined in the future.

\section{REFERENCES}

[1] N. Ihara and N. Hamada, Seikatsu Eisei, 54, 304-311 (2010).

[2] H. Kourai, Hyomen Kagaku, 22, 663-670 (2001).

[3] H. Kanazawa, The Adhesion Society of Japan, 46, 151$157(2010)$

[4] K. Takeda, K. Endoh, T. Kanou and K. Sano, Marine Engineering, 50, 119-124 (2015).

[5] N. Kawashima, Journal of Synthetic Organic Chemistry, 61, 496-505 (2003).

[6] T. Okabe and K. Saitou, Mokuzai Hozon, 19, 66-76 (1993).

[7] T. Okabe, H. Ono and S. Kodate, Journal of Japan Association on Odor Environment, 43, 128-137 (2012).

[8] S. Anant, B. Brian, K. Joanna and Padmaja S, Cellulose, 14, 65-71 (2006).

[9] W. Yaming, X. Yanmei, Y. Dongran, Y. Wen, Y. Chuntai and S. Changyu, Materials Letters, 128, 85-88 (2014).

[10] JIS L 1902 (2015).

[11] Society of International sustaining growth for Antimicrobial Articles, S06, 1-6 (2016).

[12] JIS K 7127 (1999).

[13] Society of International sustaining growth for Antimicrobial Articles, K07, 1-11 (2017).

[14] T. Okabe, Y. Morita, Y. Inamori, K. Narita and R. Yamamoto, Mokuzai Hozon, 32, 225-231 (2006).

[15] T. Noda and M. Shimizu, Seikatsu Eisei, 44, 13-19 (2000).

[16] A. Yasui, NIPPON GOMU KYOKAISHI, 80, 19-24 (2007).

[17] Y. Nakamura, K. Fujita, M. Adachi, Y. Kinugawa, T. Iida, M. Sasaki and Y. Urahama, Journal of The Adhesion Society of Japan, 41, 498-506 (2005).

[18] Y. Ishi, Y. Endo, T. Tani, T. Yoshioka, K. Matuda, H. Aoki, K. Numa, H. Abe, M. Tamagawa, M. Kodama, Jinko Zoki, 18, 141-144 (1989).

(Received October 30, 2018; Accepted November 15, 2018; Published Online February 1, 2019) 